\title{
Eosinophilic Granuloma of the Liver Mimicking Metastatic Liver Tumor: A Case Report
}

\author{
Yotaro Uchida ${ }^{1}$, Keiji Yokoyama ${ }^{1}$, Tomotaka Higashi ${ }^{1}$, Takanori Kitaguchi ${ }^{1}$, Hiromi Fukuda ${ }^{1}$, \\ Ryo Yamauchi ${ }^{1}$, Naoaki Tsuchiya ${ }^{1}$, Atsushi Fukunaga ${ }^{1}$, Kaoru Umeda ${ }^{1}$, Kazuhide Takata ${ }^{1}$, \\ Takashi Tanaka ${ }^{1}$, Yasuaki Takeyama ${ }^{1}$, Satoshi Shakado ${ }^{1}$, Shotaro Sakisaka ${ }^{1}$, \\ Hiroyuki Hayashi $^{2}$, Yoshihiro Hamada ${ }^{2}$, Kazuki Nabeshima ${ }^{2}$ and Fumihito Hirai ${ }^{1}$
}

\begin{abstract}
:
We herein report a case of coagulation necrosis with granulation and eosinophilic infiltration of the liver. A 37-year-old woman was diagnosed with a new mass lesion in the liver 1 month after breast cancer surgery and admitted for a further examination. Because the tumor occurred immediately after surgery, it was considered essential to determine whether or not it was a metastatic liver tumor from breast cancer. A percutaneous liver tumor biopsy revealed eosinophilic granuloma of the liver, which is considered to have a high possibility of visceral larva migrans with suspected gnathostomiasis infection. A detailed medical history and histological diagnosis are important for making a differential diagnosis.
\end{abstract}

Key words: Liver tumor, eosinophilic granuloma of the liver, visceral larva migrans, gnathostomiasis infection, percutaneous biopsy

(Intern Med Advance Publication)

(DOI: 10.2169/internalmedicine.8438-21)

\section{Introduction}

Eosinophilic granuloma of the liver (EGL) is caused by chronic inflammation, such as that due to larva migrans, chronic bacterial liver abscess, tuberculosis, fungi, necrotizing vasculitis, sarcoidosis, or drug use (1-8). The most common subjective symptom is abdominal pain, but it is not uncommon for patients to have no symptoms. Blood test results are characterized by elevated eosinophil and immunoglobulin E (IgE) levels (1). However, it is often difficult to make a clear diagnosis of EGL based solely on imaging findings, and a liver tumor biopsy is often useful for making a definitive diagnosis.

We herein report a case of coagulation necrosis with granulation and eosinophilic infiltration of the liver with characteristic histological findings that was diagnosed by a liver tumor biopsy.

\section{Case Presentation}

\section{Patient information and clinical findings}

A 37-year-old woman visited our hospital for the evaluation of a liver tumor. The patient had undergone left mastectomy and a sentinel lymph node biopsy for left breast cancer (stage II) at a local doctor in December X-1. The lymph node metastasis had been $\leq 2 \mathrm{~mm}$ in size and seen at a single location, so axillary lymph node dissection had been omitted, with follow-up continued. In January X, 1 month after breast cancer surgery, she had been diagnosed with a new mass lesion (diameter, approximately $30 \mathrm{~mm}$ ) in segment 5 of the liver after undergoing magnetic resonance imaging (MRI) for postoperative follow-up, and she was admitted to our hospital for a further examination.

The patient showed no significant symptoms upon admission. Her vital signs were stable. She had no history of alco-

${ }^{1}$ Department of Gastroenterology and Medicine, Fukuoka University Faculty of Medicine, Japan and ${ }^{2}$ Department of Pathology, Fukuoka University Faculty of Medicine, Japan

Received: August 1, 2021; Accepted: September 1, 2021; Advance Publication by J-STAGE: October 19, 2021

Correspondence to Dr. Yokoyama Keiji, yokotin@fukuoka-u.ac.jp 
Table. Laboratory Data on Admission.

\begin{tabular}{lclc}
\hline Hematology values & & \multicolumn{2}{l}{ Blood chemistry values } \\
WBC count & $5,700 / \mu \mathrm{L}$ & TP & $7.6 \mathrm{~g} / \mathrm{dL}$ \\
$\quad$ Neutrophil & $56.5 \%$ & Alb & $4.8 \mathrm{~g} / \mathrm{dL}$ \\
Eosinophil & $5.2 \%$ & T-bil & $0.7 \mathrm{mg} / \mathrm{dL}$ \\
Basophil & $2.1 \%$ & AST & $24 \mathrm{U} / \mathrm{L}$ \\
$\quad$ Monophil & $4.7 \%$ & ALT & $17 \mathrm{U} / \mathrm{L}$ \\
$\quad$ Lymphocyte & $31.5 \%$ & LDH & $142 \mathrm{U} / \mathrm{L}$ \\
RBC count & $475 \times 10^{4} / \mu \mathrm{L}$ & ALP & $194 \mathrm{U} / \mathrm{L}$ \\
Hemoglobin & $12.3 \mathrm{~g} / \mathrm{dL}$ & GGT & $13 \mathrm{U} / \mathrm{L}$ \\
Hematocrit & $41.3 \%$ & ChE & $306 \mathrm{U} / \mathrm{L}$ \\
Platelet count & $39.9 \times 10^{4} / \mu \mathrm{L}$ & BUN & $11 \mathrm{mg} / \mathrm{dL}$ \\
Coagulation & & Cre & $0.54 \mathrm{mg} / \mathrm{dL}$ \\
PT & $106 \%$ & eGFR & $90.4 \mathrm{~mL} / \mathrm{min}$ \\
INR & 1.02 & Hepatitis viral marker \\
APTT & $27.8 \mathrm{~s}$ & HBsAg & - \\
Fibrinogen & $344 \mathrm{mg} / \mathrm{dL}$ & HBcAb & - \\
Serology & & HCVAb & - \\
IgG & $1,415 \mathrm{mg} / \mathrm{dL}$ & Tumor marker \\
IgA & $165 \mathrm{mg} / \mathrm{dL}$ & CEA & $<1.8 \mathrm{ng} / \mathrm{mL}$ \\
IgM & $199 \mathrm{mg} / \mathrm{dL}$ & CA19-9 & $19 \mathrm{U} / \mathrm{mL}$ \\
IgE & $252 \mathrm{mg} / \mathrm{dL}$ & AFP & $<2.0 \mathrm{ng} / \mathrm{mL}$ \\
ANA & $<40 \mathrm{Dil}$ & DCP & $19 \mathrm{mAU} / \mathrm{mL}$ \\
& & CA15-3 & $8.3 \mathrm{U} / \mathrm{mL}$ \\
& & &
\end{tabular}

Ab: antibody, ANA: Anti-nuclear antibody, AFP: $\alpha$-fetoprotein, Ag: antigen, ALB: albumin, ALP: alkaline phosphatase, ALT: alanine transaminase, APTT: activated partial thromboplastin time, AST: aspartate transaminase, BUN: blood urea nitrogen, CA15-3: carbohydrate antigen 15-3, CEA: carcinoembryonic antigen, ChE: cholinesterase, Cre: creatinine, DCP: des- $\gamma$-carboxy prothrombin, eGFR: estimated glomerular filtration rate, GGT: $\gamma$-glutamyl transpeptidase, $\mathrm{HBc}$ : hepatitis B core, $\mathrm{HBV}$ : hepatitis B virus, $\mathrm{HCV}$ : hepatitis C virus, $\operatorname{IgA}$ : immunoglobulin A, IgE: immunoglobulin E, IgG: immunoglobulin G, IgM: immunoglobulin M, INR: international normalized ratio, L3: lectin3, LDH: lactate dehydrogenase, PT: prothrombin time, RBC: red blood cell, Tbil: total bilirubin, TP: total protein, WBC: white blood cell

hol consumption or smoking. She had an allergic reaction to amoxicillin and clarithromycin (a rash appeared). Laboratory investigations revealed an increase in the platelet count, eosinophil ratio, and IgE level. No abnormalities were found in the coagulation system or general biochemical tests. Serologic markers for hepatitis $\mathrm{B}$ and $\mathrm{C}$ were negative. Regarding tumor markers, the patient's total $\alpha$-fetoprotein, des- $\gamma$ carboxy prothrombin, carcinoembryonic antigen, carbohydrate antigen 19-9, and carbohydrate antigen 15-3 levels were normal (Table).

Abdominal ultrasonography showed a hypoechoic lesion measuring approximately $30 \mathrm{~mm}$ in segment 5 of the liver (Fig. 1a). In addition, two linear echoes were found inside the hypoechoic lesions (Fig. 1b). Abdominal contrastenhanced computed tomography (CE-CT) revealed a hypovascular tumor with a faint contrast effect on the margin and a diameter of $28 \mathrm{~mm}$ at segment 5 of the liver (Fig. 2a-c). At the same nodule site, abdominal contrastenhanced MRI revealed a tumor showing a low signal on T1-weighted imaging and non-uniform high signal on T2weighted imaging. Diffusion-weighted imaging showed an indistinct high signal, and the apparent diffusion coefficient mapping image was mildly high. On contrast-enhanced imaging, the surrounding area of the nodule was stained in a ring shape in the dynamic phase, and the entire nodule was defective in the hepatobiliary phase (Fig. 3a-f). In addition, positron emission tomography (PET) revealed a mild abnormal accumulation of fluorodeoxyglucose (FDG) (early maximum standardized uptake value $=2.92$, delayed maximum standardized uptake value=2.98) (Fig. 4a, b).

\section{Progress after hospitalization}

Breast cancer histopathology revealed a luminal A-like pattern (negative for the human epidermal growth factor receptor type 2, high expression of the hormone receptor, low proliferative capacity, and low grade). The histological findings were low for malignancy and considered unlikely to lead to distant metastasis; however, since the liver tumor was a new lesion that occurred after breast cancer surgery, it was considered essential to determine whether or not it was a metastatic liver tumor from breast cancer, so a percutaneous tumor biopsy was performed. The target nodule was extremely elastic and hard, making it difficult for the biopsy needle to advance into the nodule, but tumor tissue was ultimately able to be collected.

Histopathological results showed coagulative necrotizing nodules with marked eosinophil infiltration (Fig. 5a, b), granulation tissue (Fig. 6a), and some Langhans giant cells in the nodule (Fig. 6b). Immunostaining was negative for gross cystic disease fluid protein-15, mammaglobin, and estrogen receptor (Fig. 7a-c) and positive for only cytokeratin7 in the glandular duct where a non-neoplastic glandular duct existed (Fig. 7d). Therefore, liver metastasis of breast cancer was considered negative. In addition, Ziehl-Neelsen (Fig. 7e), Gram, Grocott, and periodic acid Schiff-Alcian Blue staining did not confirm any pathogens, such as bacteria, tubercle bacilli, fungi, or amoeba. Furthermore, no evidence of vasculitis was observed by Elastica van Gieson staining.

The pathological diagnosis was coagulation necrosis with granulation and eosinophilic infiltration. In this patient, there were some typical images suggesting granulomatous inflammation; for example, Langhans giant cells were observed, and eosinophil infiltration in the tissue was also clear, suggesting the possibility of parasite-related lesions. In the serum parasite antibody qualitative screening test performed to determine the cause, the gnathostomiasis antibody was Class 1, suggesting an early or chronic stage of infection. Subsequent re-collection of her life history revealed that she had a history of having eaten raw carp at a travel destination just before her breast cancer surgery, suggesting a potential infection route.

\section{Outcome and follow-up}

The liver nodule on abdominal ultrasonography showed a shrinking tendency and became obscure over the natural course of the infection (Fig. 8a-c), and the gnathostomiasis 


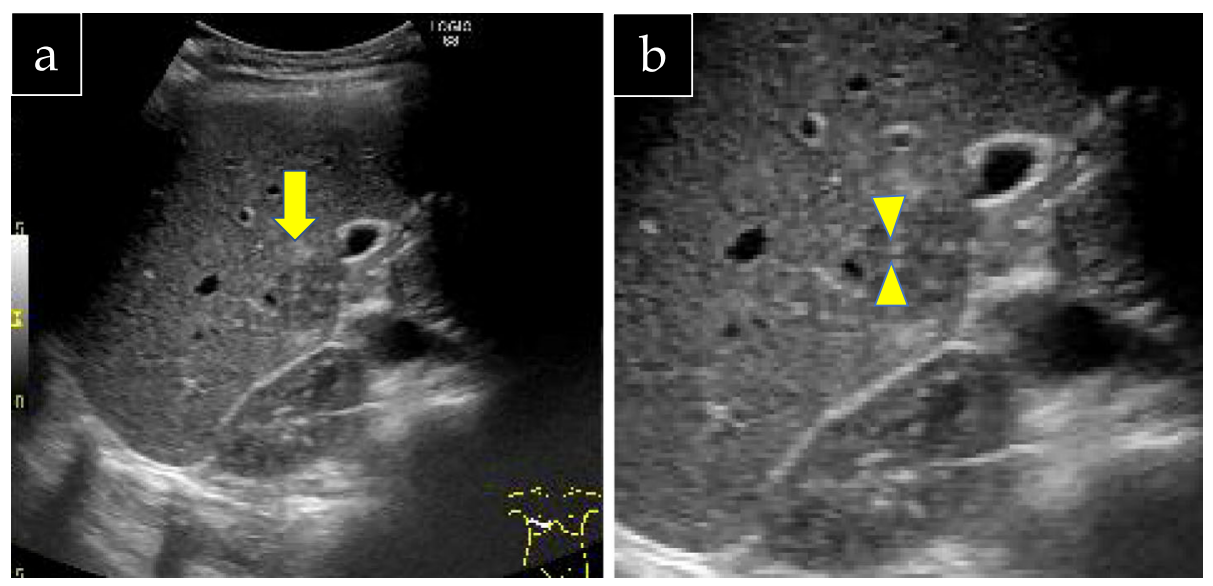

Figure 1. Abdominal ultrasonographic imaging findings. (a) Hypoechoic lesion in segment 5 of the liver (arrow). (b) Two linear echoes inside the nodule (arrowhead; bead sign).
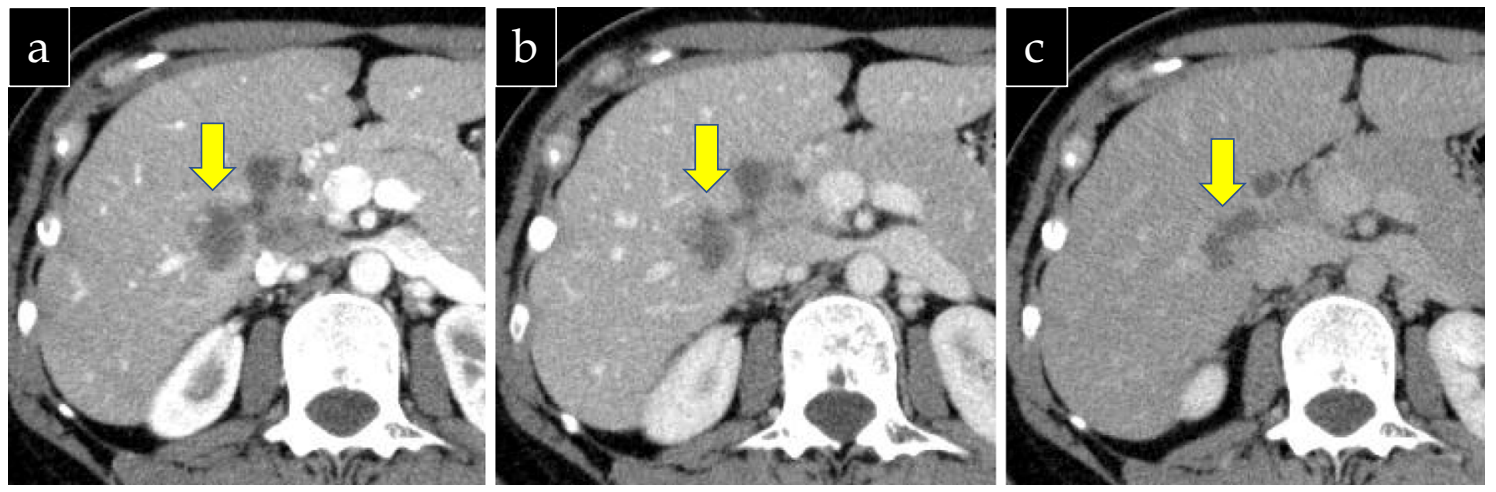

Figure 2. Abdominal contrast-enhanced computed tomography imaging findings of the nodule in segment 5 of the liver. (a) Early phase (arrow). (b) Portal phase (arrow). (c) Late phase (arrow).
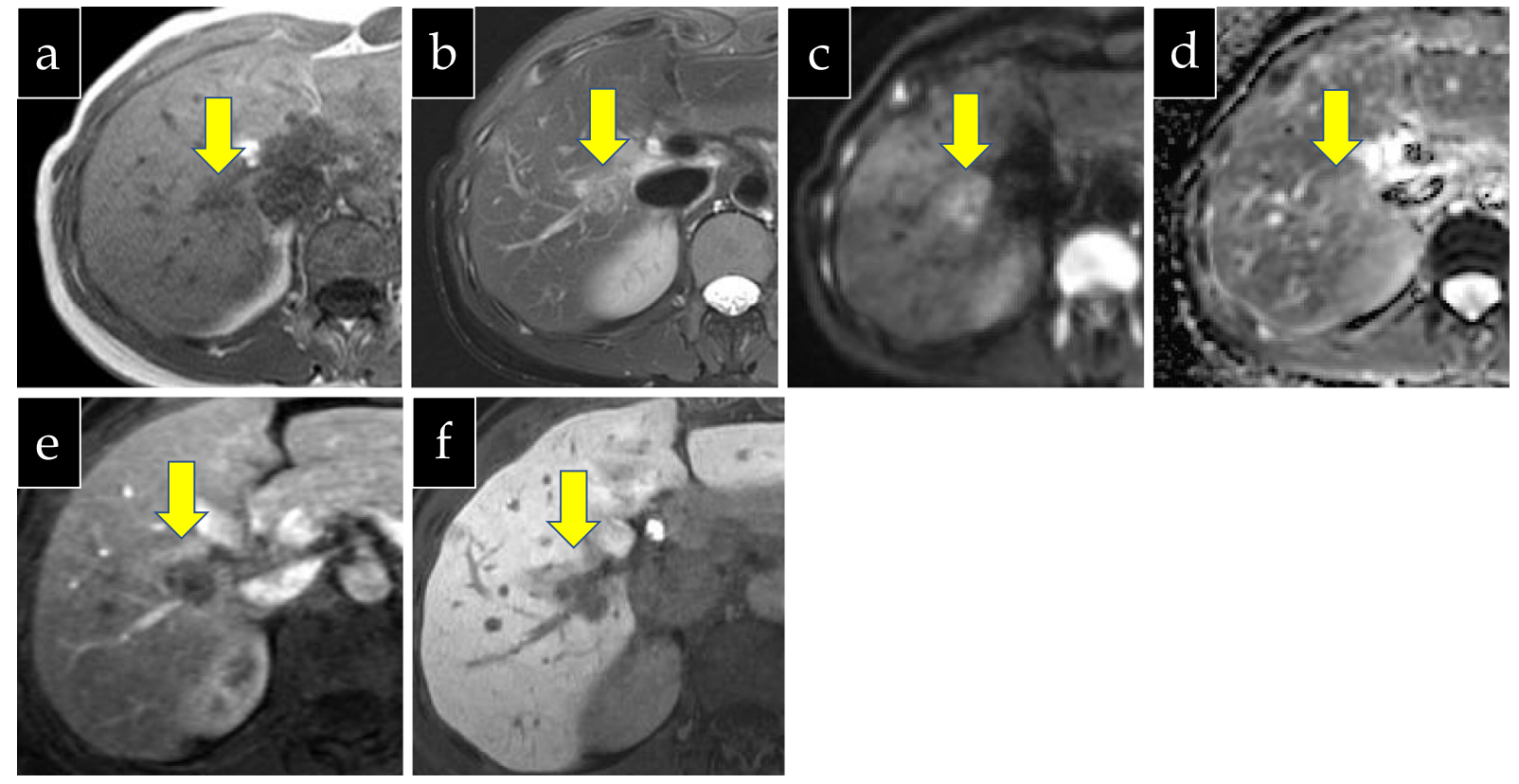

Figure 3. Abdominal contrast-enhanced magnetic resonance image findings of the nodule in segment 5 of the liver. (a) T1-weighted imaging (arrow). (b) T2-weighted imaging (arrow). (c) Diffusionweighted imaging (arrow). (d) Apparent diffusion coefficient mapping image (arrow). (e) Dynamic phase (arrow). (f) Hepatobiliary phase (arrow). 

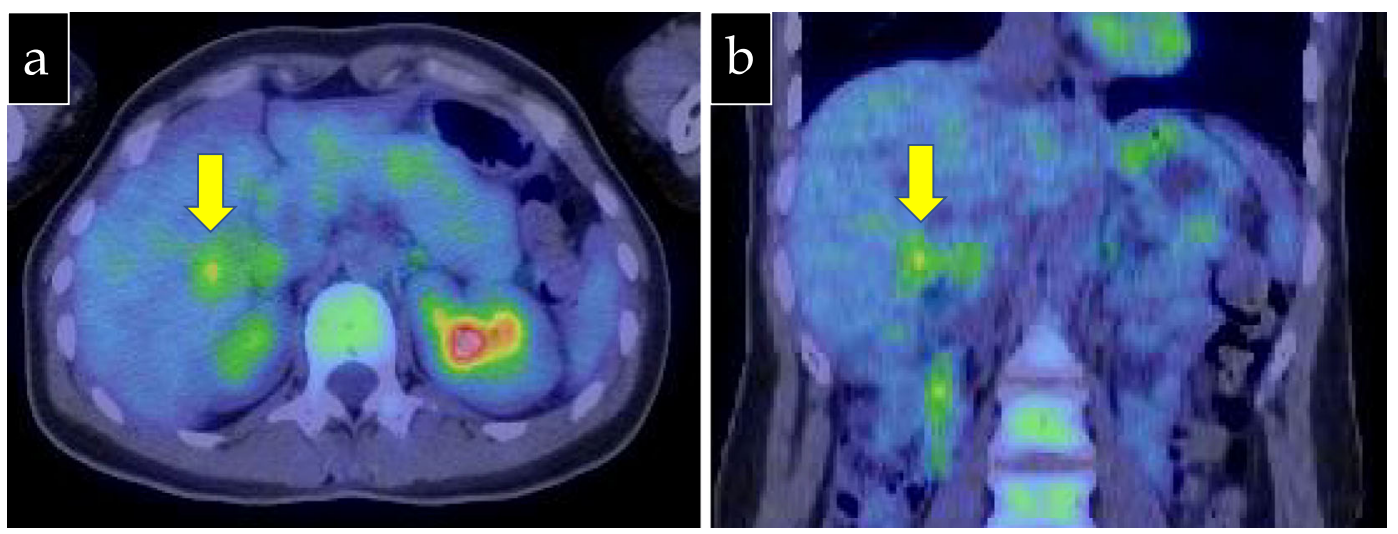

Figure 4. Positron emission tomography imaging findings of the nodule in segment 5 of the liver. The accumulation of FDG in the axial view (arrow). (b) FDG accumulation in the coronal view (arrow). FDG: fluorodeoxyglucose

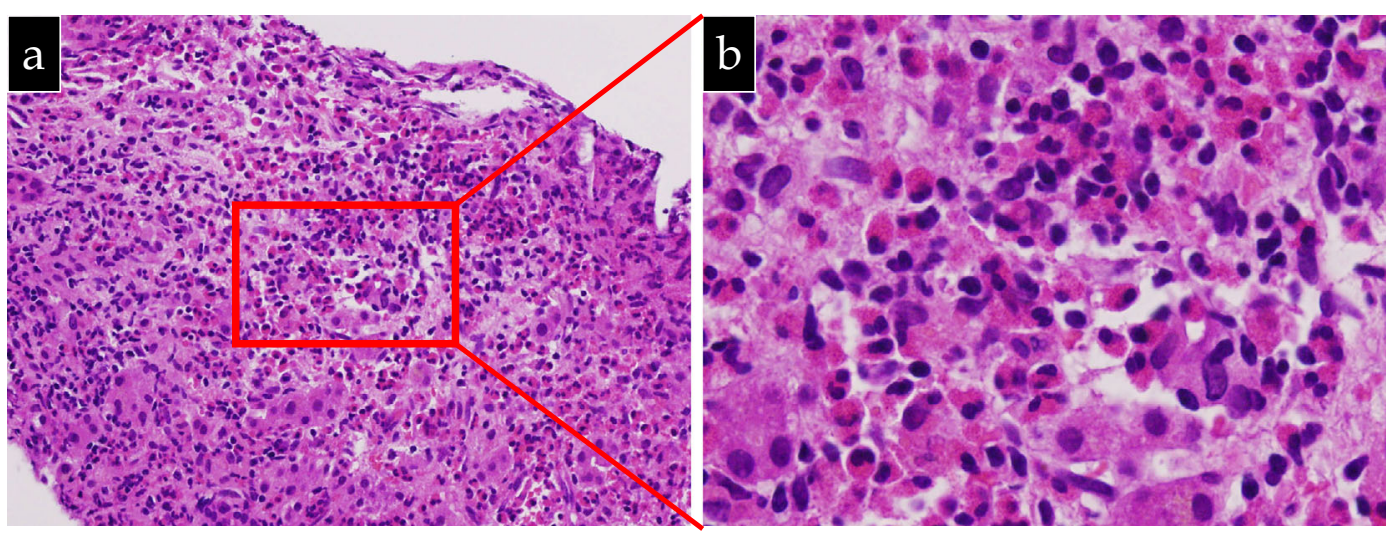

Figure 5. Histopathological results of the nodule in segment 5 of the liver. The coagulative necrotizing nodule with marked eosinophil infiltration. (a) Findings at $100 \times$ magnification. (b) Findings at $600 \times$ magnification; the part surrounded by the red frame in (a) is magnified.
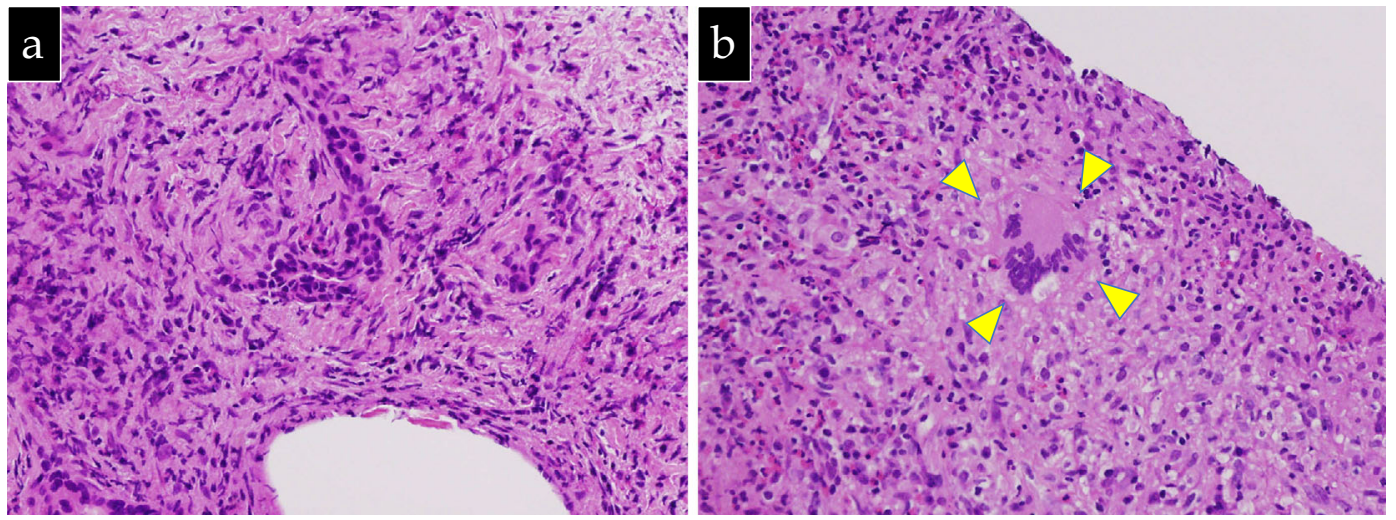

Figure 6. Histopathological results of the nodule in segment 5 of the liver (findings at 200x magnification). (a) The coagulative necrotizing nodule with granulation tissue. (b) Langhans giant cells in the nodule (arrowhead).

antibody qualitative test became negative six months later. In addition, the eosinophil ratio decreased from $5.2 \%$ to $2.4 \%$, and the IgE level decreased from 252 to $167 \mathrm{mg} / \mathrm{dL}$. She is currently being followed up on an outpatient basis.

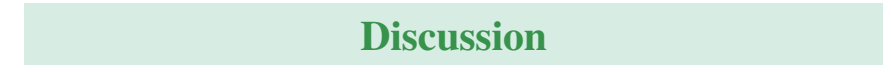

The possibility of EGL caused by type I allergy-induced antigens, such as chronic bacterial liver abscess, tuberculo- 

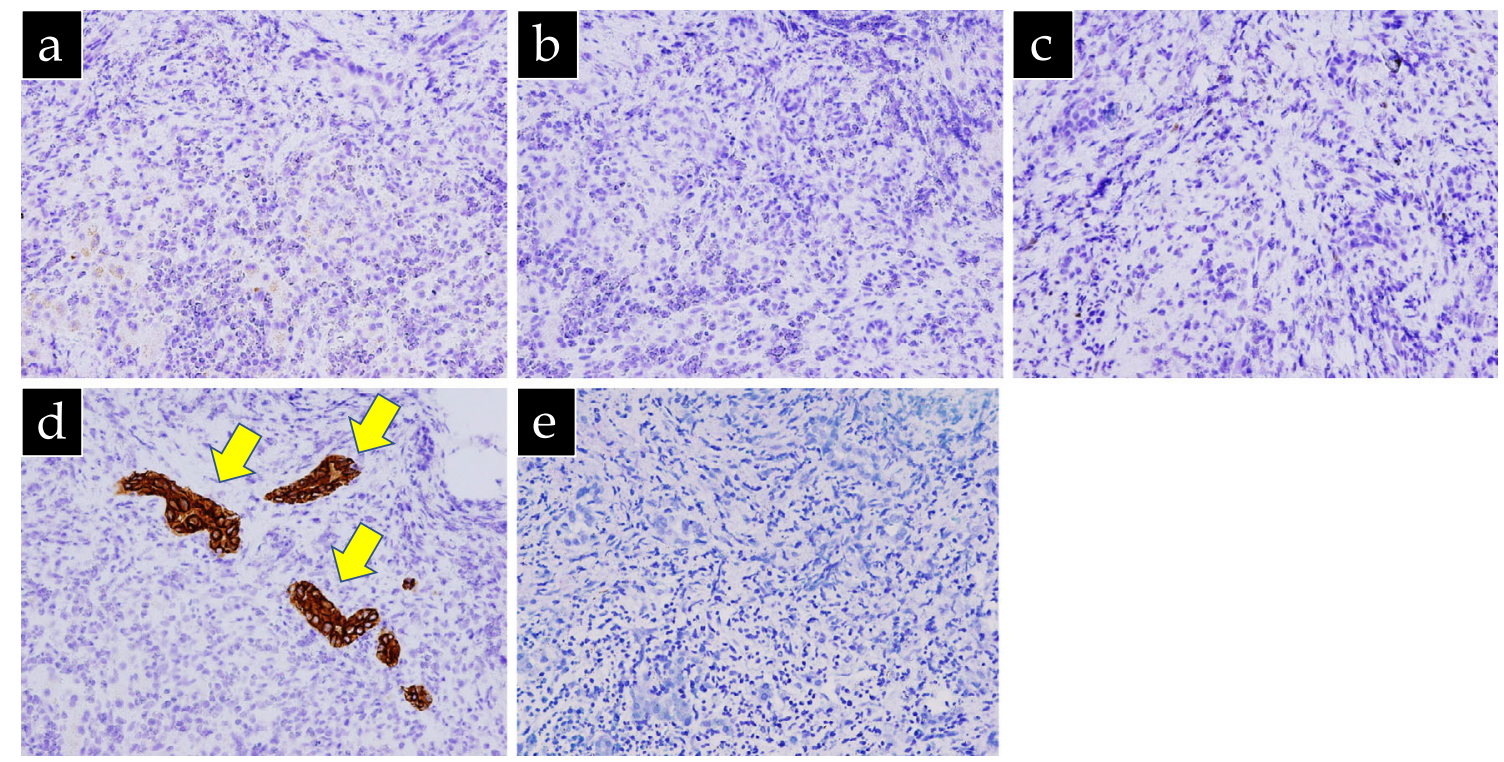

Figure 7. Immunostaining findings of the nodule in segment 5 of the liver (findings at $200 \times$ magnification). (a) Negative for gross cystic disease fluid protein-15. (b) Negative for mammaglobin. (c) Negative for the estrogen receptor. (d) Positive for only cytokeratin-7 (arrow) in the glandular duct where a non-neoplastic glandular duct exists. (e) Negative for the Ziehl-Neelsen.
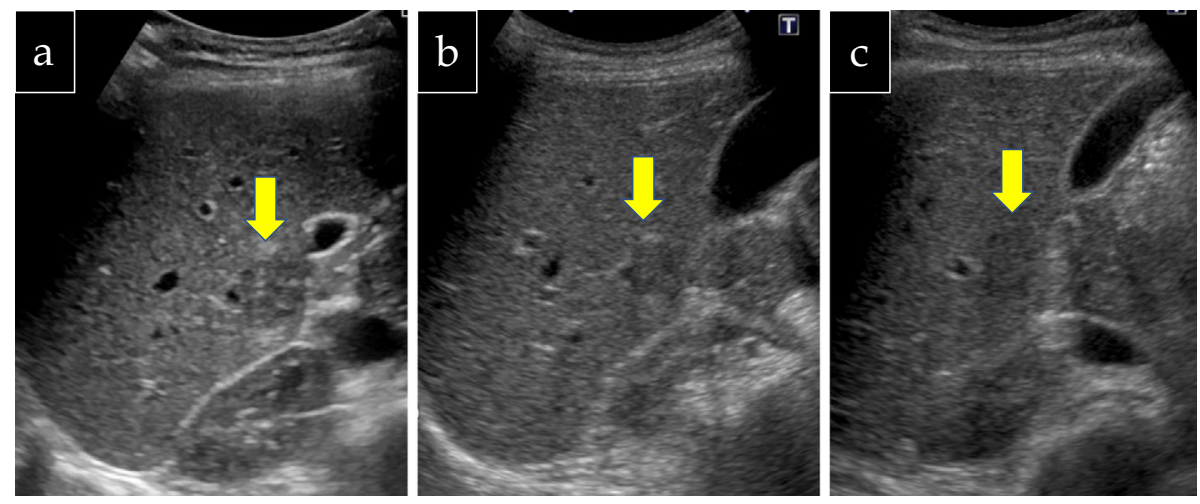

Figure 8. Follow-up of the nodule in segment 5 of the liver. The liver nodule tended to shrink and become obscure. (a) At the time of the liver biopsy (arrow). (b) Three months later (arrow). (c) Six months later (arrow).

sis, fungi, necrotizing vasculitis, sarcoidosis, and drug use (mentioned in the introduction), could not be completely ruled out in the present case due to the difficulty of making a definitive diagnosis. However, based on the medical history and serum antibody qualitative test results, this case was considered to have a high possibility of involving visceral larva migrans (VLM).

VLM is the most common cause of EGL; however, it is often not possible to confirm the parasite body. Indeed, Kaplan et al. reported that $65 \%$ of 43 cases of EGL had no parasite bodies (9). Since there are many cases in which a diagnosis is made based on the medical history and serum antibodies, as in this case, it is necessary to continue to accumulate cases in which careful listening of the medical history and various tests, such as serum antibodies have been performed.

Larvae that cause VLM-related liver granulomatous nod- ules include Schistosoma japonicum, Clonorchis sinensis, Fasciola hepatica, dog/cat/pig roundworm, fecal nematode, tapeworm, Anisakis, and gnathostomiasis (10-17), and sources of infection include pet breeding and eating raw meat from cattle, chicken, and river fish. However, people may incidentally become infected with such eggs either excreted or spread by dogs and cats in sandboxes and park pathways. In addition, a person's exact history of previously consuming raw meat of fish is often not clear. As a result, it is often difficult to identify the cause of such infections.

In the present case, gnathostomiasis was Class 1 according to the parasite antibody qualitative screening test. $\mathrm{Hu}-$ man gnathostomiasis is most frequently caused by the nematode Gnathostoma spinigerum. This disease is endemic to Southeast Asia, particularly Thailand and Japan. Infection has been reported to occur from consuming raw freshwater fish, such as black bass, raigyo, carp, and catfish as well as 
snakes (18). In the human body, the parasite moves under the skin as a larva, causing a linear repelling rash of the skin and migratory edema. Occasionally, invasion of the liver, eyes, nerves, spinal cord, and brain has been reported (17). Most patients heal spontaneously within two to three months, and indeed, in the present case, the liver nodules tended to become obscure and shrink over the natural course of the infection.

The characteristics of image inspection were compared with those of previous reports (19-24); abdominal ultrasonography often shows a single lesion on the liver surface visualized as having clear boundaries and a hypoechoic nodule. The bead sign, which is two linear echoes that emphasize the portal vein branch in the granuloma, was suspected in this case (Fig. 1b). This sign is characteristic of granulomas with eosinophilic infiltrate and is found in about $70 \%$ of cases $(25,26)$.

In the current case, a faint but dark stain around the area was observed in the early phase of CE-CT, which was consistent with previously reported findings (19-21). On MRI, the tumor lumen often shows a low signal on T1-weighted imaging and a medium to high signal on T2-weighted imaging. In our case, T1-weighted imaging revealed a low signal, while T2-weighted imaging showed a faint high signal, which was consistent with previously reported findings $(19,22)$. In addition, PET-CT revealed the mildly abnormal accumulation of FDG. There have been several reports of hepatic granuloma showing the accumulation of FDG on PET-CT, similar to this patient $(23,24)$. However, these findings may not be typical, as the imaging features vary depending on the stage.

Several cases in which the imaging findings of VLM syndrome mimic malignant lesions have been reported $(27,28)$. In addition, owing to its non-specific appearance on CT, hepatic toxocariasis can often be mistaken for other conditions. Differential diagnoses for multiple low-density liver nodules include microabscesses, other granulomatous diseases (i.e. sarcoidosis), and hepatocellular carcinoma or liver metastasis (29). Hepatic lesions associated with VLM usually appear as multiple ill-defined, oval-shaped, low-attenuating nodules that are best seen in the portal phase on CT. These findings seem to be somewhat characteristic of eosinophilic infiltration or granuloma and can thus distinguish such processes from malignant lesions. Histologic confirmation is one of the most important ways of making a differential diagnosis.

The pathological findings showed granulation tissue and coagulative necrosis, and eosinophil-based inflammatory cell infiltration was characteristic, which was consistent with this case. Although Charcot-Leyden crystals were not observed in this case, about half of the cases of VLM have diamondshaped crystals that stain red with the growth of collagen fibers that crystallize after degranulation of eosinophil granules. This is a useful characteristic finding $(30,31)$.

In conclusion, in the present patient, although it was difficult to determine whether or not this case involved a metas- tatic liver tumor from breast cancer, a percutaneous tumor biopsy revealed a coagulative necrotic nodule with granulation and eosinophilic infiltration that was suspected to be VLM. Collecting a detailed medical history and obtaining a histological diagnosis are important for making a differential diagnosis.

The authors state that they have no Conflict of Interest (COI).

\section{Acknowledgments}

We would like to thank Editage (www.editage.com) for the English language editing. We also would like to express our gratitude to Eri Yamauchi, Motoko Kawashima, Waki Nagashima, Yuki Nozaki, Atsuko Ishibashi, Rina Akahoshi, Chihiro Tanaka, Akiko Tanaka, and Tomoko Nagaura for their invaluable support.

\section{Consent and confidentiality}

Written informed consent was obtained from the patient for publication of this manuscript. Patient identifiers, aside from age and sex, were removed, and the images provided were anonymized to protect patient confidentiality.

\section{References}

1. Irani SK, Dobbins WO 3rd. Hepatic granulomas: review of 73 patients from one hospital and survey of the literature. J Clin Gastroenterol 1: 131-143, 1979.

2. Turhan N, Kurt M, Ozderin YO, Kurt OK. Hepatic granulomas: a clinicopathologic analysis of 86 cases. Pathol Res Pract 207: 359365, 2011.

3. Coash M, Forouhar F, Wu CH, Wu GY. Granulomatous liver diseases: a review. J Formos Med Assoc 111: 3-13, 2012.

4. Sanai FM, Ashraf S, Abdo AA, Satti MB, Batwa F, Al-Husseini $\mathrm{H}$. Hepatic granuloma: decreasing trend in a high-incidence area. Liver Int 28: 1402-1407, 2008.

5. Charatcharoenwitthaya $\mathrm{P}$, Apisarnthanarak P, Pongpaibul A, Boonyaarunnate T. Eosinophilic pseudotumour of the liver. Liver Int 32: 311, 2012.

6. Gambari PF, Ostuni PA, Lazzarin P, Fassina A, Todesco S. Eosinophilic granuloma and necrotizing vasculitis (Churg-Strauss syndrome?) involving a parotid gland, lymph nodes, liver and spleen. Scand J Rheumatol 18: 171-175, 1989.

7. Israel HL, Margolis ML, Rose LJ. Hepatic granulomatosis and sarcoidosis. Further observations. Dig Dis Sci 29: 353-356, 1984.

8. McMaster KR 3rd, Hennigar GR. Drug-induced granulomatous hepatitis. Lab Invest 44: 61-73, 1981.

9. Woodruff AW. Toxocariasis. Brit Med J 3: 663-669, 1970.

10. Beaver PC. The nature of visceral larva migrans. J Parasital 55: 3$12,1969$.

11. Shimokawa H. Visceral larva migrans by Toxocara cati. Fukuoka Acta Med 73: 64-69, 1982.

12. Schantz PM. Toxocaral visceral larva migrans. New Engl J Med 298: 436-439, 1978.

13. Hayashi K, Tahara H, Yamashita K, et al. Hepatic imaging studies on patients with visceral larva migrans due to probable Ascaris suum infection. Abdom Imaging 24: 465-469, 1999.

14. Vercelli-Retta J, Lagios MD, Chandrasoma P. Fasciola hepatica and parasitic eosinophilic granuloma of the Liver. Am J Surg Pathol 26: 1238, 2002.

15. Saito F, Okabe Y, Suga H, Watanabe T, Arinaga T, Naito Y. A case of hepatic eosinophilic granuloma, which needs distinction with metastatic liver cancer. J Jpn Soc Gastroenterol 105: 1509-1514, 
2008.

16. Liu GH, Sun MM, Elsheikha HM, et al. Human gnathostomiasis: a neglected food-borne zoonosis. Parasit Vectors 13: 616, 2020.

17. Kaplan KJ, Goodman ZD, Ishak KG. Eosinophilic granuloma of the liver: a characteristic lesion with relationship to visceral larva migrans. Am J Surg Pathol 25: 1316-1321, 2001.

18. Rusnak JM, Lucey DR. Clinical gnathostomiasis: case report and review of the English-language literature. Clin Infect Dis 16: 33 50, 1993.

19. Lim JH. Toxocariasis of the liver: visceral larva migrans. Abdom Imaging 33: 151-156, 2008.

20. Chang S, Lim JH, Choi D, et al. Hepatic visceral larva migrans of Toxocara canis: CT and sonographic findings. AJR Am J Roentgenol 187: W622-W629, 2006.

21. Lim JH. Hepatic visceral larva migrans of Toxocara canis. Am J Trop Med Hyg 82: 520-521, 2010.

22. Azuma K, Yashiro N, Kinoshita T, Yoshigi J, Ihara N. Hepatic involvement of visceral larva migrans due to Toxocara canis: a case report--CT and MR findings. Radiat Med 20: 89-92, 2002.

23. Crucitti A, Grossi U, Leccisotti L, et al. Food residue granuloma mimicking metastatic disease on FDG-PET/CT. Jpn J Radiol 31: 349-351, 2013.

24. Kim SW, Shin HC, Kim IY, et al. Foreign body granulomas simulating recurrent tumors in patients following colorectal surgery for carcinoma: a report of two cases. Korean J Radiol 10: 313-318,
2009.

25. Grover JK, Vats V, Uppal G, Yadav S. Anthelmintics: a review. Trop Gastroenterol 22: 180-189, 2001.

26. Hirata T, Yamasaki K, Li YG, Majima Y, Tsuji M. Demonstration of hepatic granuloma due to visceral larva migrans by ultrasonography. J Clin Ultrasound 18: 429-433, 1990.

27. Lim YJ, Kim JH, Oh SH, Jeon SC, Koh HC, Lee YH. Pulmonary toxocariasis masquerading as metastatic tumor nodules in a child with osteosarcoma. Pediatr Blood Cancer 53: 1343-1345, 2009.

28. Kang EJ, Choi YJ, Kim JS, et al. Bladder and liver involvement of visceral larva migrans may mimic malignancy. Cancer Res Treat 46: 419-424, 2014.

29. Hossack J, Ricketts P, Te HS, Hart J. A case of adult hepatic toxocariasis. Nat Clin Pract Gastroenterol Hepatol 5: 344-348, 2008.

30. Thakral D, Agarwal P, Saran RK, Saluja S. Significance of Charcot Leyden crystals in liver cytology-A case report. Diagn Cytopathol 43: 392-394, 2015.

31. $\mathrm{Su}$ J. A Brief History of Charcot-Leyden Crystal Protein/Galectin10 Research. Molecules 23: 2931, 2018.

The Internal Medicine is an Open Access journal distributed under the Creative Commons Attribution-NonCommercial-NoDerivatives 4.0 International License. To view the details of this license, please visit (https://creativecommons.org/licenses/ by-nc-nd/4.0/).

\section{(C) The Japanese Society of Internal Medicine} Intern Med Advance Publication 